\title{
Are electronic cigarettes and vaping effective tools for smoking cessation? Limited evidence on surgical outcomes: a narrative review
}

\author{
Amber Famiglietti ${ }^{1}$, Jessica Wang Memoli ${ }^{2}$, Puja Gaur Khaitan ${ }^{1,3} \wedge$ \\ ${ }^{1}$ Department of Surgery, Georgetown University School of Medicine, MedStar Washington Hospital Center, Washington, D.C., USA; ${ }^{2}$ Division of \\ Interventional Pulmonology, Georgetown University School of Medicine, MedStar Washington Hospital Center, Washington, D.C., USA; ${ }^{3}$ Division \\ of Thoracic and Esophageal Surgery, Georgetown University School of Medicine, MedStar Washington Hospital Center, Washington, D.C., USA \\ Contributions: (I) Conception and design: A Famiglietti, PG Khaitan; (II) Administrative support: None; (III) Provision of study materials or patients: \\ None; (IV) Collection and assembly of data: All authors; (V) Data analysis and interpretation: A Famiglietti, PG Khaitan; (VI) Manuscript writing: \\ All authors; (VII) Final approval of manuscript: All authors. \\ Correspondence to: Puja Gaur Khaitan, MD, FACS. Associate Professor, Department of Surgery, Division of Thoracic and Esophageal Surgery, \\ Georgetown University School of Medicine, MedStar Washington Hospital Center; 110 Irving Street, NW (G253); Washington, D.C. 20010, USA. \\ Email: Puja.G.Khaitan@medstar.net.
}

\begin{abstract}
The rising popularity of e-cigarettes and vaping, particularly in youth populations, has prompted the scientific community to ocassionally recommend their use as alternative to smoking or as a modality for smoking cessation. Media also tends to portray them as stylish, smoking cessation tools. We first studied the current literature to better understand whether they are viable options for surgeons to use prior to surgery as part of their armamentarium and their efficacy in attaining smoking abstinence. Next, we performed a comprehensive review of the literature to study the impact of e-cigarette and vaping on lung pathophysiology, surgical outcomes, and postoperative complications. After a thorough search, we found limited evidence suggesting that e-cigarettes and vaping are effective smoking cessation tools, and indeed may increase the propensity of dual smoking, contrary to e-cigarette advertisements. Many potential biases and limitations exist due to self-reporting when investigating e-cigarettes and vaping. While there is controversial data in the literature about e-cigarettes and vaping not leading to lung cancer, there are chemicals in these products that compromise lung hemostasis, negatively affect the immune system, and have detrimental inflammatory effects on wound healing. Studies are warranted to elucidate objective data regarding short and long-term effects of these products on surgical outcomes, and given the current data, they should not be utilized as viable smoking cessation tools.
\end{abstract}

Keywords: Electronic cigarettes; vaping; smoking cessation; surgical outcomes

Submitted Jul 26, 2020. Accepted for publication Nov 12, 2020.

doi: $10.21037 /$ jtd-20-2529

View this article at: http://dx.doi.org/10.21037/jtd-20-2529

$\wedge$ ORCID: 0000-0001-8654-062X. 


\section{Introduction}

Electronic cigarettes (e-cigarettes) are marketed as both a healthy alternative to traditional tobacco products and as a useful smoking cessation tool. The popularity of e-cigarettes has increased over the past two decades while the percentage of Americans that smoke tobacco cigarettes has continued to decline. In $1998,25.9 \%$ of males and $22.1 \%$ of females 18 or older smoked tobacco cigarettes in the United States, compared to $16 \%$ of males and $12.3 \%$ of females in 2017 (1). Per the CDC National Youth Tobacco Survey, tobacco cigarette use in individuals ages 17 and younger dropped from $35.1 \%$ in 1999 to $6.9 \%$ in 2014 (2). In stark contrast, noncombustible product use such as e-cigarettes and chewing tobacco, increased in the same demographics from $4.3 \%$ to $30.2 \%$ in 1999 and 2014, respectively (2). Notably, an upward trend was observed in 2011 with dual use of cigarette and noncombustible products, as well as poly-use, which is defined as cigarette use, noncombustible use, and consumption of non-cigarette combustibles (i.e., cigars, pipes, and hookah) (2). This rise has been a cause for concern, as multiple studies hypothesize that e-cigarettes may function as a gateway to youth use of drugs other than nicotine, such as cannabis $(3,4)$. Furthermore, additional research efforts investigating outcomes of e-cigarette use on patient health are warranted.

In this review, we first discuss the history of e-cigarette use, its wide adoption, and its documented effects on pulmonary homeostasis, lung function, and risk of lung cancer, followed by a focused review on its effectiveness as a smoking cessation tool and the resultant short and long-term effects of its use on postoperative complications. We present the following article in accordance with the narrative review checklist (available at http://dx.doi.org/10.21037/jtd-20-2529).

\section{Methods}

Multiple sources were used for this overview including Medline, EMBASE, and Cochrane database. We performed a comprehensive search to understand the impact of e-cigarette use and vaping on lung pathophysiology and looked for literature that could elucidate any postoperative complications associated with their use. Given that the new generation often uses these products as smoking cessation tactic, we also searched to determine the efficacy of e-cigarettes and vaping in attaining smoking abstinence. During our search, we particularly looked for systematic reviews, meta-analysis, and level of evidence on these subjects.

\section{History of electronic cigarettes and their growing popularity}

The origin of e-cigarettes dates back to 1965 when Herbert Gilbert patented the first "smokeless nontobacco cigarette" in the United States (Figure 1) (5). This product was developed as an alternative to tobacco cigarettes, as evidence emerged in the 1960s linking tobacco smoking to lung cancer (5). Gilbert's battery-powered device delivered heated flavor elements like rum, mint, and cinnamon without combustion or the use of tobacco and nicotine; it quickly became popular but never emerged in commercial markets. Next, in 1986, the "Favor cigarette" emerged as another noncombustible device advertised as an alternative to nicotine containing products but was also unsuccessful (5). In 2003, Chinese pharmacist Hon Lik received support from investors for mass production of his electronic atomizing cigarette in the Chinese market under the company name Ruyan (5). This device was marketed as a smoking cessation tool and alternative to tobacco cigarettes. Widespread advertising and internet marketing contributed to the rapid entrance of this product into U.S. markets in 2006 (5).

E-cigarettes create an inhaled aerosol or "vapor" by rapidly heating a customizable liquid, rather than by combustion as in conventional tobacco cigarettes. Their popularity has continued to increase over the past decade. E-cigarette use, defined in one study as use within the past 30 days or ever-use in one's lifetime more than tripled in 2016 compared to 2011 in middle and high school aged students, and more than doubled in young adults aged 18-24 (5). Another study using a database of participating retailers found that the overall sales of e-cigarettes increased from $\$ 11.6$ million in 2010 to $\$ 751.2$ million in 2016 (6). The price of both rechargeable and disposable electronic devices has declined over time, as the tobacco industry bought out many brands and grew to control $72.7 \%$ of the market in 2016 compared to a complete absence of ownership in 2010 (6).

In addition to decreasing cost and increasing accessibility, another attraction of these devices is customizability (7). The consumer is able to pick from a variety of devices to deliver the e-cigarette liquid known as e-fluid or e-juice and choose the level of nicotine and flavor (Figure 2). Immense popularity has promoted large scale development, 


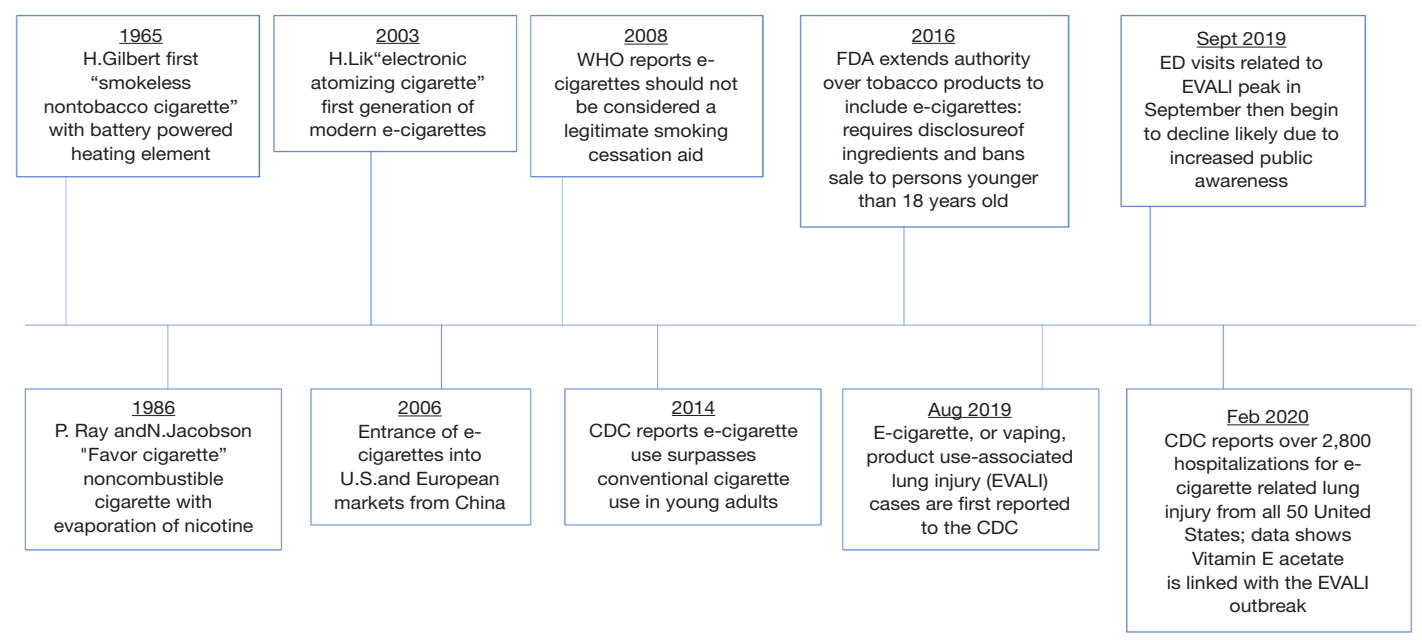

Figure 1 Historical timeline of the introduction of e-cigarettes and subsequent notable events.

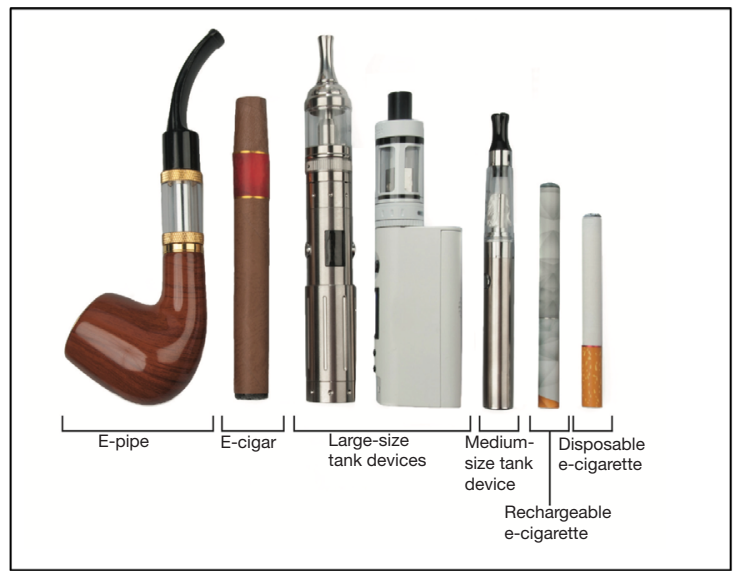

\begin{tabular}{|c|c|c|}
\hline Product & Description & Some brands \\
\hline Disposable e-cigarette & $\begin{array}{l}\text { Cigarette-shaped device consisting of a battery and } \\
\text { a cartridge containing an atomizer to heat a solution } \\
\text { (with or without nicotine). Not rechargeable or } \\
\text { refillable and is intended to be discarded after } \\
\text { product stops producing vapor. Sometimes called } \\
\text { an e-hookah. }\end{array}$ & $\begin{array}{l}\text { NJoY OneJoy, Aer } \\
\text { Disposable, } \\
\text { Flavorvapes }\end{array}$ \\
\hline Rechargcable e-cigarette & $\begin{array}{l}\text { Cigarettc-shaped device consisting of a battery that } \\
\text { connects to an atomizer used to heat a solution } \\
\text { typically containing nicotine. Often contains an } \\
\text { element that regulates punf duration and/or how } \\
\text { many puffs may be taken consecutively. }\end{array}$ & $\begin{array}{l}\text { Blu,GreenSmoke, } \\
\text { EonSmoke }\end{array}$ \\
\hline Pen-style, medium-sized rechargcable e-cigarette & $\begin{array}{l}\text { Larger than a cigarette, often with a higher-capacity } \\
\text { battery, may contain a prefilled cartridge or a } \\
\text { refilable cartridge. Often come with a manual } \\
\text { switch allowing the user to regulate length and } \\
\text { frequency of puffs. }\end{array}$ & $\begin{array}{l}\text { Vapor King Storm, } \\
\text { Totally Wicked } \\
\text { Tornado }\end{array}$ \\
\hline Tank-style, large-sized rechargeable e-cigarette & $\begin{array}{l}\text { Much larger than a cigarette with a higher-capacity } \\
\text { battery and typically contains a large, refillable } \\
\text { cartridge. Often contains manual switches and a } \\
\text { battery casing for customizing battery capacity.Can } \\
\text { be easily modified. }\end{array}$ & Volcano Lavatube \\
\hline
\end{tabular}

Figure 2 The e-cigarette epidemic. Various forms of e-cigarette products currently available ranging from an elite-appearing cigar to more metallic vaping devices with colorful products to more cig-alike e-cigarettes as shown in both panels above. Image on left obtained from ref (8); image on the right obtained from ref (9). Prepared for World Health Organization Tobacco Free Initiative. December 2013. Accessed Apr 2, 2020. Reprinted with permission from UCSF.

including a wide variety of thousands of flavors with more than 460 brands of e-cigarettes available (10). In the U.S. market, JUUL has become the most popular brand of e-cigarettes with disposable pods, now accounting for more than half of the e-cigarette retail market share (11). In a survey of current or ever e-cigarette users in high school, these products were found to be the most popular, which is concerning given the high nicotine content of JUUL e-cigarette fluid compared to other e-cigarette brands $(12,13)$. These often-rechargeable electronic devices with refillable and disposable cartridges continue to become more advanced and generate smaller particles with more effective nicotine delivery (7), thus potentially making them more addictive (Figure 3).

In December 2019, the President signed the Tobacco 21 legislation raising the minimum age for sale of tobacco and e-cigarette products from 18 to 21 years old (15). The increase in regulation came at a time when studies showed increasing levels of adolescent e-cigarette use and advertisements targeting this population (Figure 4). One study found the prevalence of ever use of e-cigarettes to be highest in individuals age 18 to 34 , even after stratification by 

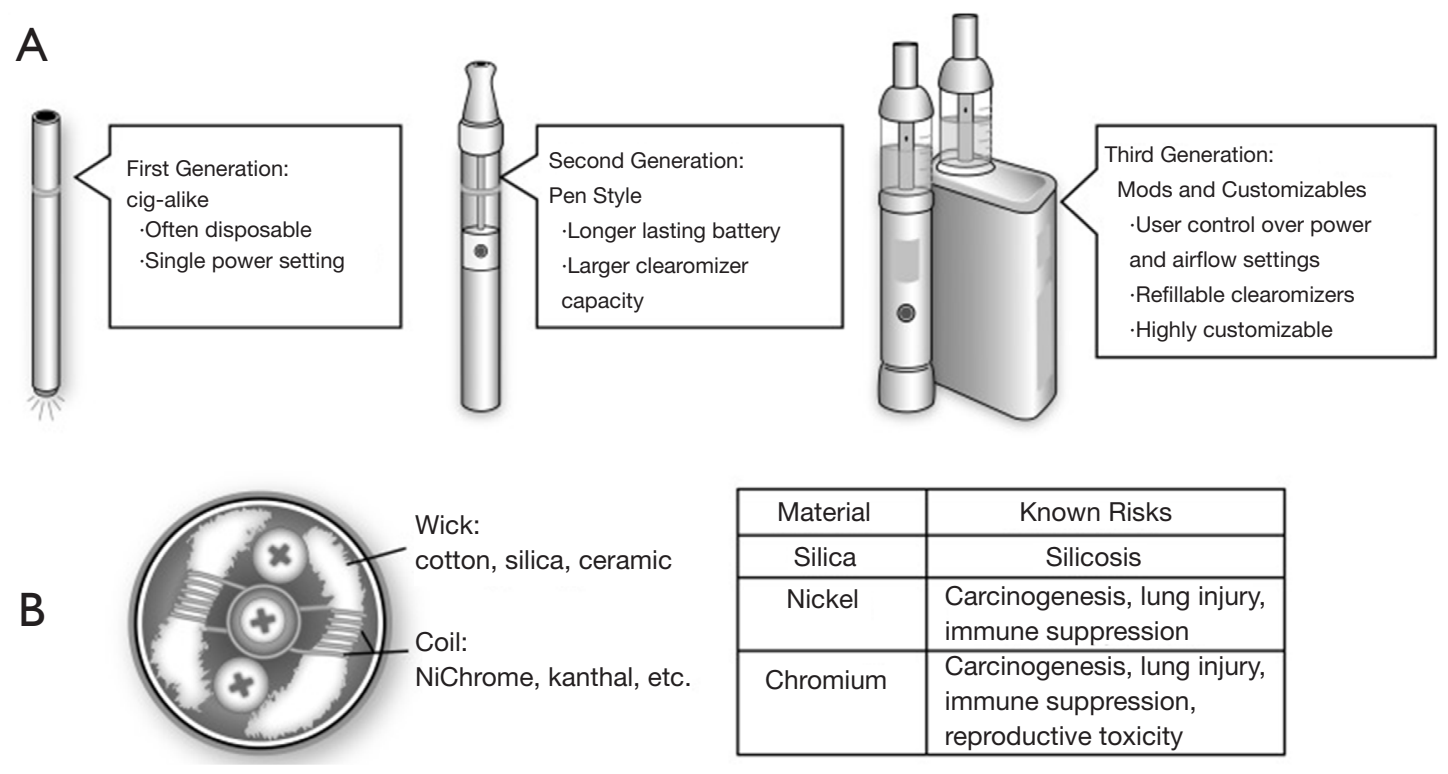

\begin{tabular}{|c|l|}
\hline Material & \multicolumn{1}{|c|}{ Known Risks } \\
\hline Silica & \multicolumn{1}{|c|}{ Silicosis } \\
\hline Nickel & $\begin{array}{l}\text { Carcinogenesis, lung injury, } \\
\text { immune suppression }\end{array}$ \\
\hline Chromium & $\begin{array}{l}\text { Carcinogenesis, lung injury, } \\
\text { immune suppression, } \\
\text { reproductive toxicity }\end{array}$ \\
\hline
\end{tabular}

Figure 3 The evolution of electronic cigarettes. (A) Illustration of first, second and third generation electronic cigarettes. While e-cigarettes started out as a cig-alike devices, they have evolved into more user-friendly tools with the customer's ability to control the resistance of aerosolization process and subsequent effect. (B) The wick and coil components of the atomizers in e-cigarettes harbor various chemicals with known risks of pulmonary toxicity. Permission (14) to reprint granted by The American Physiological Society.
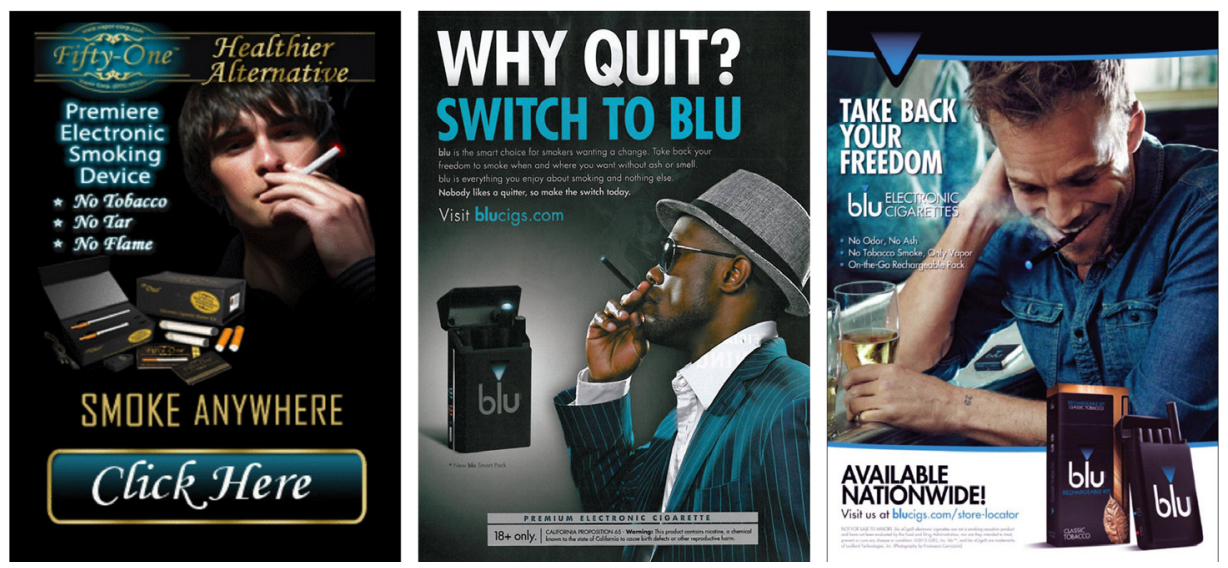

Figure 4 Various advertisements for electronic cigarettes and vaping devices portraying them as suitable smoking cessation tools, including writing logos such as "Nobody likes a quitter; so make the switch today" and "Take back your freedom". From the collection of Stanford Research into the Impact of Tobacco Advertising (tobacco.stanford.edu).

concurrent cigarette smoking status (16). A higher likelihood of ever e-cigarette use was also found in individuals with a lower education level attained, incomes below the poverty level, and with individuals that are separated, widowed, or divorced (16). The increasing popularity of these devices among youth was also exemplified by a $10 \%$ increase in amount of $12^{\text {th }}$ grade youth vaping observed between 2017 and 2018 in the U.S. (17).

The growing popularity of e-cigarettes may contribute to the social acceptance of traditional cigarette smoking as "e-cig" devices simulate tobacco products and often contain nicotine. One study examining the prevalence of e-cigarette use found that the majority of current e-cigarette users are also concurrent tobacco cigarette users $(68.1 \%)$ or former 
smokers (23.7\%) (18). Albeit, there is a growing number of never-smokers who are also adopting e-cigarettes in order to "fit-in" (8.2\%) (18).

\section{Electronic cigarette use, vaping, and lung disease}

The growing popularity of e-cigarettes has prompted the medical community to investigate the potentially adverse side effects of e-cigarette use on lung pathophysiology and lung neoplasms. Tobacco cigarettes create an inhaled aerosol via combustion that generates carcinogens, toxins and oxidizing agents (7). E-cigarettes or imitators of traditional tobacco cigarettes, conversely, create an inhaled aerosol or "vapor" by rapidly heating a customizable liquid containing propylene glycol or glycerin, nicotine, and flavoring agents (7). Furthermore, the use of e-cigarettes is called "vaping", and e-cigarettes may also be referred to as electronic nicotine delivery systems (ENDS), vapor pens, and vape pipes. It is important to note that both tobacco and e-cigarettes can effectively deliver nicotine, an addictive compound, to the body.

Use of e-cigarettes has also been studied in humans, importantly in patients with chronic obstructive pulmonary disease (COPD). Ever having used an e-cigarette was associated with worse outcomes in patients with or at risk for COPD, as demonstrated by increased prevalence of chronic bronchitis after adjusting for current age, race, and tobacco pack-year history $(\mathrm{P}<0.001)(19)$. This study found no association between e-cigarette use and smoking cessation or reduction, nor tobacco cigarette use (19). Additionally, patients with COPD who switch from traditional cigarettes to electronic devices do not demonstrate immediate improvement or decline in respiratory function quantified by $\% \mathrm{FEV} / \mathrm{FVC}$, but do experience improved subjective and objective disease outcomes such as physical activity tolerance and number of exacerbations over time (20). It is important to note that this is not uncommon in COPD patients after quitting smoking or with irreversible lung disease and one cannot exclude these findings as simply a result of tobacco smoking cessation rather than a benefit of e-cigarettes. Another study showed worsened inflammatory effects after e-cigarette use in mild asthmatics compared to non-asthmatics (21). Total respiratory impedance calculated by pressure-flow ratios and total airway resistance was significantly higher in the asthma group compared to nonasthmatics at all time points studied (21). Indeed, GarciaArcos et al. neatly demonstrated that when healthy mice were exposed daily to nicotine-containing e-cig solution over a 4-month period, they exhibited increased airway hyper-reactivity, mucin production, cytokine expression and distal airspace enlargement, all characteristics associated with COPD (22). Of note, these effects were nicotine dependent and mice exposed to nicotine free e-solution did not exhibit these deleterious effects (22).

Recent outbreaks of pulmonary disease in young adults in the United States have called attention to the deleterious effects of e-cigarettes. In a case study examining an 18-year-old patient, e-cigarette use was associated with hypersensitivity pneumonitis and acute respiratory distress syndrome requiring intubation and pressor support, which rapidly improved with steroid treatment (23). Recent outbreaks of severe pulmonary disease associated with e-cigarette use have been described. From June 2019 to August 2019, a study in Wisconsin and Illinois identified 53 patients who developed syndromic pulmonary disease with bilateral infiltrates on chest imaging (24). Pulmonary infection was ruled out in these patients, and they had all used e-cigarettes within 90 days of symptom onset (24). Of these patients, $94 \%$ were hospitalized, 32\% intubated and one died; median patient age was 19 years old and $84 \%$ of patients used tetrahydrocannabinol (THC) in their e-cigarette devices (24). A follow up nation-wide investigation spearheaded by the CDC found 805 cases of lung injury associated with e-cigarettes among 46 states and one U.S. territory (25). Among the 514 patients able to provide a detailed history, $76.9 \%$ used THC containing e-devices and $56.8 \%$ used nicotine-containing products (25). To date, the chemical exposure causing this outbreak is being actively investigated. A recent study showed vitamin $\mathrm{E}$ acetate, an additive of many THC products, to be strongly associated with e-cigarette, or vaping, product use-associated lung injury (EVALI) (26). In essence, this is a true epidemic that is impacting the health of our younger generation and if not addressed early, will impact our healthcare system and the newer generations to come.

\section{Electronic cigarettes and the COVID-19 pandemic}

The recent emergence of the coronavirus disease (COVID-19) pandemic caused by severe acute respiratory syndrome coronavirus 2 (SARS-CoV-2) has generated immense international concern as over 6 million confirmed cases and 300,000 global deaths have been documented through June, 2020 (27). Angiotensin-converting enzyme 2 (ACE2), a receptor commonly expressed in the pulmonary and 
cardiovascular systems has been established as the SARS$\mathrm{CoV}-2$ receptor, which the virus uses to gain cellular entry at the epithelial surface $(28,29)$. Increased levels of ACE2 gene expression have been found in tobacco smokers compared to non-smokers, which may account for greater susceptibility of the smoking population to COVID-19 (30). Similar to other respiratory infections, smoking is associated with adverse outcomes and increased severity of COVID-19 disease (31-34). Additionally, tobacco smoking is often associated with the most prevalent comorbid health conditions among patients hospitalized with COVID-19, such as hypertension, cardiovascular disease, diabetes, and COPD $(32,35)$.

To date, limited data exists describing the relationship between e-cigarette smoking and COVID-19 disease outcomes. Although tobacco smoking has been shown to increase ACE2 expression, e-cigarette use did not increase ACE2 expression in a study examining bronchial epithelial cells (36). However, flavor/nicotine-containing e-cigarette use was associated with dysregulation of cytokines and upregulation of inflammasome related genes, potentially exacerbating COVID-19-related inflammation (36). Hence, the healthcare and scientific community are recommending cessation of all types of smoking devices during this pandemic while further investigations are under way (31,37-39). As described above, e-cigarette use is associated with significant pulmonary toxicity and inflammation, which likely enhances symptomology and respiratory disease severity in COVID-19 patients. A recent study examining adolescents and young adults found e-cigarette users have a five-fold increased risk of COVID-19 diagnosis compared to a seven-fold increased risk of diagnosis in dual tobacco and e-cigarette users, compared to non-users $(40,41)$. The sharing of ENDS and repetitive hand-to-mouth movements while vaping increases an individual's risk of viral contamination (31). Additional research examining the impact of e-cigarette use on COVID-19 patient outcomes is warranted. In particular, a retrospective population-based analysis of exposure to e-cigarette use and COVID-19 patient outcomes will help answer some of these questions.

\section{Electronic cigarettes and cancer}

Many studies examine the link between e-cigarettes and cancer. We found e-cigarette use to cause significant cell toxicity and dysregulation of cancer-related genes. E-cigarettes have the potential to deliver decreased, yet significant, levels of carcinogens compared to conventional cigarettes (42). Formaldehyde, acetaldehyde, acrolein, carcinogenic nitrosamines $\mathrm{N}^{\prime}$-nitrosonornicotine (NNN) and nicotine-derived nitrosamine ketones (NNK) were found in vapors of a variety of e-cigarette products and are all carcinogenic to humans; heavy metals such as cadmium, nickel and lead have also been reported (43). In a study comparing tobacco cigarette use, dual tobacco and e-cigarette use, nicotine replacement therapy alone, and e-cigarette use alone for at least a 6-month period found similar overall nicotine intake among groups and equivalent levels of carcinogens or toxins in the e-cigarettes group compared to nicotine replacement therapy alone (including nicotine gum and patches) (42). E-cigarette and nicotine replacement therapy (NRT) groups had significantly lower levels of toxins and carcinogenic metabolites than combustible cigarette, dual combustible cigarette/NRT and dual combustible cigarette/e-cigarette groups (42).

Another study found current non-tobacco-smoking, e-cigarette users to have significantly higher urinary levels of the carcinogenic compounds o-toluidine and 2-naphthylamine than current non-smoking, non-e-cigarette controls; however, this study was limited by a small sample size and history of conventional cigarette use in all experimental and some control participants (44). It is important to note that exposure to both o-toluidine and 2-naphthylamine has been implicated in the development of bladder cancer from studies utilizing industrial workers $(45,46)$.

E-cigarette extracts have also been shown to play a role in cancer cell transcription factor activation. Specifically, e-cigarette extracts have been shown to induce expression of SOX2, an embryonic stem cell factor required for maintenance of stem cell properties in non-small cell lung cancer (47). In contrast, a separate study demonstrated that exposure to e-cigarette vapor resulted in no significant increase of in vitro expression of mutagenic reporter genes $c I I$ and SUPF in mouse and human fibroblast cells, respectively (48). An initiation step of metastatic disease, the epithelial to mesenchymal transition, occurred in lung cancer cells exposed to e-cigarette liquids or aerosols (49). In terms of bystander exposure to these products, one study found a five-fold higher excess life cancer risk of second hand exposure to tobacco cigarette smoke compared to e-cigarette vapors (50).

\section{Electronic cigarettes as a smoking cessation tool}

There are several health concerns that one must be 
aware of before recommending the use of e-cigarettes as a smoking cessation tool. While e-cigarettes have been used to help patients quit, there is no clear consensus on their efficacy in smoking cessation (51). No difference in quit rates or reduced number of cigarettes smoked were found at 6 months between nicotine and non-nicotine containing e-cigarettes in small treatment groups with an overall quit rate of $15 \%$ (52). Importantly, only half of the individuals that quit continued to use e-cigarettes at the follow up period of 6 months (52). A recent systematic review including 13 studies found no significant increase in smoking cessation in e-cigarette users compared to nonusers (53).

A longitudinal study of patients from 2013-2015 demonstrated that former cigarette smokers who began using e-cigarettes daily were 5.7 times more likely to reduce average daily cigarette use by $50 \%$ and 7.88 times more likely to quit smoking for at least 30 days compared to non-electronic cigarette users (54). Participants who used e-cigarettes sporadically or on some days of the week did not exhibit a significant reduction in smoking frequency or cessation; a major limitation of this study, similar to others, was the use of self-reporting (54). In contrast, another study associated e-cigarette smoking to an increased frequency of daily tobacco cigarette smoking use (55). In a survey of over 12,000 individuals, dual e-cigarette and tobacco cigarette users smoked 15.6 cigarettes/day compared to non-electronic cigarette users who smoked 14.4 cigarettes/ day $(\mathrm{P}<0.05)(55)$. Those that used nicotine e-cigarettes had a lower likelihood of being former conventional cigarette smokers compared with individuals that had never used nicotine e-cigarettes (55). A meta-analysis including 20 studies demonstrated a $28 \%$ lower likelihood of smoking cessation among individuals using e-cigarettes compared to non-electronic cigarette users, independent of the smokers' intention to quit (56). Another problematic finding is the increased consumption of alcohol in individuals that transition from tobacco use to e-cigarette use (57). This may be attributed to the social pairing of alcohol consumption with e-cigarette use.

A randomized controlled trial published in New England Fournal of Medicine in 2019 found one-year smoking abstinence rates to be significantly higher in patients randomized to nicotine containing e-cigarettes group (18\%) versus NRT (9.9\%) for smoking cessation (58). Common side effects were nausea in NRT group compared to mouth or throat irritation in e-cigarette users (58). Furthermore, the success of the e-cigarette group may be due to persistent use and longer duration of treatment as only $9 \%$ of participants continued NRT at one year compared to $80 \%$ of continued use in the e-cigarette group (58). As we are aware, most patients are not only addicted to nicotinecontaining products but are also addicted to the habit of smoking.

Given that nicotine is an addictive substance and low levels of carcinogens are present in NRT, the use of FDAapproved bupropion and varenicline may be a superior alternative for patients attempting to achieve long-term abstinence from tobacco smoking. A Cochrane review including over 100,000 participants found varenicline to be superior to a single form of NRT and bupropion alone in helping participants quit (59). Equal efficacy was observed between NRT and bupropion in smoking cessation (59). Adverse events varied by treatment: participants in the NRT group experienced orodental problems, gastrointestinal disturbances, and skin irritation (patch only) compared to nausea, insomnia, and headaches in the varenicline group, and insomnia, dry mouth, nausea and, rarely, seizures in the bupropion group (59).

A few studies describe the use of e-cigarettes as a tool to reduce perioperative tobacco cigarette use, as a recent survey in Australia found there is a general perception from surgeons and anesthesiologists that e-cigarettes and vaping can be used as a smoking alternative (60-62). Furthermore, because there is no clear literature linking poor surgical outcomes to e-cigarettes and vaping, clinicians have reported both optimism and skepticism to recommend e-cigarettes as a tool for smoking cessation prior to surgery. This gap in knowledge justifies future large-scale studies that must be conducted to provide health authorities and clinical societies the data needed to establish universal guidelines regarding the use of e-cigarettes and vaping as a smoking cessation tool.

\section{Electronic cigarettes and surgery}

There is a severe paucity of data on the impact of e-cigarette use on surgical outcomes and wound healing. One such study examined skin flap survival using a rat model exposed to e-cigarettes in a smoking chamber, where nicotine containing e-cigarettes were found to negatively impact skin flap survival and tissue perfusion $(63,64)$. When compared to controls, significantly higher rates of dorsal skin flap necrosis were found in rats exposed to nicotine containing e-cigarettes and tobacco cigarettes $(63,64)$. Of note, similar rates of dorsal skin flap toxicity and necrosis were found 


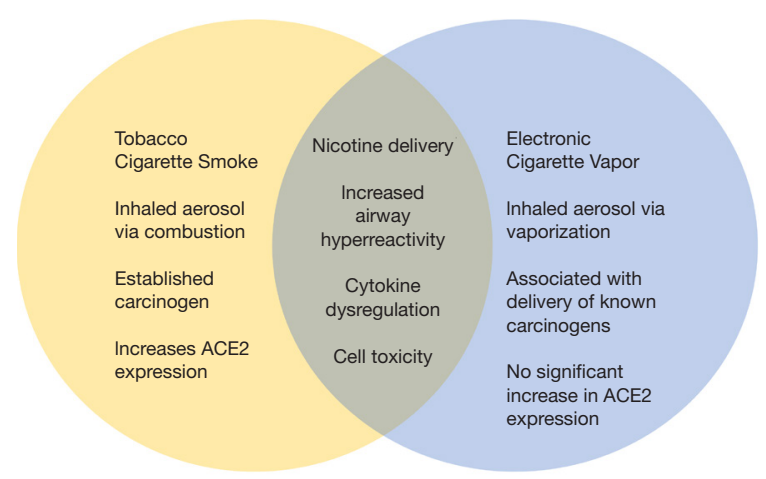

Figure 5 Summarized pictorial depicting the deleterious effects of conventional cigarette use and electronic cigarette use/vaping.

between e-cigarette and tobacco smoke exposed groups, illustrating the deleterious effects of nicotine exposure on wound healing $(63,64)$. Tissue hypoxia was also significantly higher in e-cigarette and tobacco cigarette exposed groups compared to non-exposed control rats (63).

Interestingly, one systematic review demonstrated that while nicotine appears to have detrimental effects on inflammation in wound models, there is no clear evidence to suggest any detrimental or beneficial effects on postoperative outcome of wound or tissue healing (65). In fact, there are no large studies describing any deleterious effects of e-cigarette use on wound healing, cardiovascular events, or pulmonary complications in humans. After a thorough literature search, surgical outcomes associated with e-cigarette use is limited to a small number of case studies. We found one case study describing a woman with a 25 pack-year tobacco smoking history who switched to e-cigarettes three months preoperatively and experienced significant skin flap necrosis and breast reconstruction failure after undergoing bilateral mastectomy with immediate tissue expander reconstruction for breast cancer (66). In another case study, perioperative e-cigarette use was associated with post-operative vasospasm (67). In this study, a woman undergoing bilateral mastectomy with transverse rectus abdominis musculocutaneous (TRAM) flap reconstruction for intraductal carcinoma experienced multiple episodes of post-operative vasospasm, requiring a return to the operating room for surgical exploration of the anastomosis (66). Another study found nicotine containing e-cigarettes to compromise microcirculation compared to non-smokers and nicotine-free e-cigarettes (68). Furthermore, nicotine appears to adversely affect tissue perfusion, however, additional largescale studies are needed to fully elucidate the vascular consequences of using e-cigarettes.

\section{Summary}

As e-cigarette popularity rises, their effects on human health need to be closely examined and scrutinized (Figure 5). Many potential biases and limitations exist when investigating this often-polarizing topic. Studies on this subject are severely limited by self-reporting and recall bias. Often, e-cigarette users are concurrent tobacco and cannabis smokers, making it difficult for researchers to parse out the deleterious effects of each product. While additional observational studies are required to elucidate long-term effects of e-cigarettes on lung pathophysiology, it is clear that e-cigarettes have adverse effects on lung homeostasis and are not an effective smoking cessation tool with potential wound complications. Although limited studies describe e-cigarette use as a tool to reduce perioperative tobacco smoking use (60-62), we view this alternative with skepticism and advise our patients to quit smoking altogether.

In addition to the adverse effects of e-cigarette use on lung homeostasis described above, the recent outbreaks of e-cigarette, or vaping, product use-associated lung injury (EVALI) in our youth populations, and the preliminary data describing worse outcomes in COVID-19 patients who smoke, prompts us to suggest a ban on e-cigarettes. In India, a ban on production, sale, and importation of ENDS is already in place in an effort to safeguard the younger population and thwart nicotine addiction (69). E-cigarette bans were imposed in certain states including Massachusetts, Michigan, New York, and Rhode Island after the vaping-associated lung disease emerged in fall of 2019, however, these bans were only temporary (70). We suggest implementation of a ban on sale and importation of ENDS in the United States as a public health priority. This would expand upon the World Health Organization recommendations to ban e-cigarette advertising and use indoors (71).

It is important to note that while e-cigarettes contain various carcinogenic compounds and have been shown to play a role in dysregulated carcinogenesis and lung homeostasis, there have been no direct associations made between e-cigarette use and incidence of lung cancer. We attribute this finding to lack of longitudinal studies examining ENDS and the relatively recent rise in popularity of vaping. It is also difficult to provide more comprehensive recommendations due to the inherent limitations of in vitro studies, the lack of in vivo models and the paucity of clinical data regarding e-cigarette use. To mitigate this paucity of data, surgeons and all 
clinicians alike need to specifically ask patients about and document the use of ENDS routinely, along with smoking history during patient's intake in order for us to study and understand the long-term ramifications of their use in the surgical setting. This would allow the scientific community to better understand and assess the long-term effects of e-cigarette use on incidence of end-organ damage, surgical outcomes, malignancy risk, and its validity to act as a smoking cessation tool.

With increased adoption of e-cigarettes and vaping products, it is important that clinicians carefully examine the published literature and identify knowledge gaps. Controversies are bound to persist about their use as viable smoking cessation tools for some time, until surgical outcomes are thoroughly documented and evaluated.

\section{Acknowledgments}

Jory Barone, MedStar Washington Hospital Medical Library.

Funding: None.

\section{Footnote}

Reporting Checklist: The authors have completed the Narrative Review reporting checklist. Available at http:// dx.doi.org/10.21037/jtd-20-2529

Conflicts of Interest: All authors have completed the ICMJE uniform disclosure form (available at http:// dx.doi.org/10.21037/jtd-20-2529). The authors have no conflicts of interest to declare.

Ethical Statement: The authors are accountable for all aspects of the work in ensuring that questions related to the accuracy or integrity of any part of the work are appropriately investigated and resolved.

Open Access Statement: This is an Open Access article distributed in accordance with the Creative Commons Attribution-NonCommercial-NoDerivs 4.0 International License (CC BY-NC-ND 4.0), which permits the noncommercial replication and distribution of the article with the strict proviso that no changes or edits are made and the original work is properly cited (including links to both the formal publication through the relevant DOI and the license). See: https://creativecommons.org/licenses/by-nc-nd/4.0/.

\section{References}

1. Office of Disease Prevention and Health Promotion. Tobacco Use, Adult cigarette smoking (age adjusted, percent, 18+ years) By sex [Internet]. 2020. Available online: https://www.healthypeople.gov/2020/topicsobjectives/topic/tobacco-use/national-snapshot

2. El-Toukhy S, Sabado M, Choi K. Trends in tobacco product use patterns among U.S. youth, 1999-2014. Nicotine Tob Res 2018;20:690-7.

3. Dai H, Hao J. Electronic cigarette and marijuana use among youth in the United States. Addict Behav 2017;66:48-54.

4. Dai H, Catley D, Richter KP, et al. Electronic cigarettes and future marijuana use: A longitudinal study. Pediatrics 2018;141:1-10.

5. Frieden TR, Jaffe HW, Richards CL, et al. E-Cigarette Use Among Youth and Young Adults. A Report of the Surgeon General. MMWR 2016;63:521-8.

6. Cantrell J, Huang J, Greenberg M, et al. History and Current Trends in the Electronic Cigarette Retail Marketplace in the United States: 2010-2016. Nicotine Tob Res 2020;22:843-7.

7. Glantz SA, Bareham D. E-Cigarettes: Use, Effects on Smoking, Risks, and Policy Implications. Annu Rev Public Health 2018;39:215-35.

8. E-Cigarette Use Among Youth and Young Adults. A Report of the Surgeon General. Atlanta, GA: Centers for Disease Control and Prevention, National Center for Chronic Disease Prevention and Health Promotion, Office on Smoking and Health, 2016, p.3.

9. Grana R, Benowitz N, Glantz SA. Background paper on E-cigarettes (electronic nicotine delivery systems). Center for Tobacco Control Research and Education, University of California, San Francisco, a WHO Collaborating Center on Tobacco Control.

10. Zhu SH, Sun JY, Bonnevie E, et al. Four hundred and sixty brands of e-cigarettes and counting: Implications for product regulation. Tob Control 2014;23:iii3-9.

11. Herzog B, Kanada P. Nielsen: Tobacco "All Channel" Data 1/27/2018 [Internet]. 2018. Available online: https://1bxcx1bcuig1rfxaq3rd6w9-wpengine.netdna-ssl. com/wp-content/uploads/2018/02/Nielsen-Tobacco-AllChannel-Report-Period-Ending-1.27.18

12. Krishnan-Sarin S, Jackson A, Morean M, et al. E-cigarette devices used by high-school youth. Drug Alcohol Depend 2019;194:395-400.

13. Reilly SM, Bitzer ZT, Goel R, et al. Free Radical, 
Carbonyl, and Nicotine Levels Produced by Juul Electronic Cigarettes. Nicotine Tob Res 2019;21:1274-8.

14. Chun LF, Moazed F, Calfee CS, et al. Pulmonary toxicity of e-cigarettes. Am J Physiol-Lung Cell Mol Physiol 2017;313:L193-206.

15. Tobacco 21 [Internet]. United States Food and Drug Administration. 2019. Available online: https://www.fda. gov/tobacco-products/retail-sales-tobacco-products/ tobacco-2 1

16. Stallings-Smith S, Ballantyne T. Ever Use of E-Cigarettes Among Adults in the United States: A CrossSectional Study of Sociodemographic Factors. Inquiry 2019;56:46958019864479.

17. Miech R, Johnston L, O'Malley PM, et al. Adolescent vaping and nicotine use in 2017-2018 - U.S. National estimates. N Engl J Med 2019;380:192-3.

18. Jaber RM, Mirbolouk M, Defilippis AP, et al. Electronic cigarette use prevalence, associated factors, and pattern by cigarette smoking status in the United States from NHANES (National health and nutrition examination survey) 2013-2014. J Am Heart Assoc 2018; 7:e008178.

19. Bowler RP, Hansel NN, Jacobson S, et al. Electronic Cigarette Use in US Adults at Risk for or with COPD: Analysis from Two Observational Cohorts. J Gen Intern Med 2017;32:1315-22.

20. Morjaria JB, Mondati E, Polosa R. E-cigarettes in patients with COPD: Current perspectives. Int J Chron Obstruct Pulmon Dis 2017;12:3203-10.

21. Lappas AS, Tzortzi AS, Konstantinidi EM, et al. Shortterm respiratory effects of e-cigarettes in healthy individuals and smokers with asthma. Respirology 2018;23:291-7.

22. Garcia-Arcos I, Geraghty P, Baumlin N, et al. Chronic electronic cigarette exposure in mice induces features of COPD in a nicotine-dependent manner. Thorax 2016;71:1119-29.

23. Sommerfeld CG, Weiner DJ, Nowalk A, et al. Hypersensitivity pneumonitis and acute respiratory distress syndrome from e-cigarette use. Pediatrics 2018;141:1-4.

24. Layden JE, Ghinai I, Pray I, et al. Pulmonary illness related to e-cigarette use in Illinois and Wisconsin - Final report. N Engl J Med 2020;382:903-16.

25. Perrine CG, Pickens CM, Boehmer TK, et al. Characteristics of a Multistate Outbreak of Lung Injury Associated with E-cigarette Use, or Vaping - United States, 2019. MMWR 2019;68:860-4.

26. Blount BC, Karwowski MP, Shields PG, et al. Vitamin $\mathrm{E}$ acetate in bronchoalveolar-lavage fluid associated with
EVALI. N Engl J Med 2020;382:697-705.

27. COVID-19 Dashboard by the Center for Systems Science and Engineering (CSSE) at Johns Hopkins University (JHU) [Internet]. 2019. Available online: https:// coronavirus.jhu.edu/map.html

28. Walls AC, Park YJ, Tortorici MA, et al. Structure, Function, and Antigenicity of the SARS- Structure, Function, and Antigenicity of the SARS-CoV-2 Spike Glycoprotein. Cell 2020;181:281-92.e6.

29. Yan R, Zhang Y, Li Y, et al. Structural basis for the recognition of SARS-CoV-2 by full-length human ACE2. Science 2020;367:1444-8.

30. Brake SJ, Barnsley K, Lu W, et al. Smoking Upregulates Angiotensin-Converting Enzyme-2 Receptor : A Potential Adhesion Site for Novel Coronavirus SARS-CoV-2 (Covid-19). J Clin Med 2020;9:841-8.

31. Berlin I, Thomas D, Faou A Le, et al. COVID-19 and Smoking. Nicotine Tob Res 2020;22:1650-2.

32. Zheng Z, Peng F, Xu B, et al. Risk factors of critical \& mortal COVID-19 cases: A systematic literature review and meta-analysis. J Infect 2020;81:e16-25.

33. Komiyama M, Hasegawa K. COVID-19 Smoking Cessation as a Public Health Measure to Limit the Coronavirus Disease 2019 Pandemic COVID-19. Eur Cardiol 2020;15:e16.

34. Engin AB, Engin ED, Engin A. Two important controversial risk factors in SARS-CoV-2 infection: Obesity and smoking. Environ Toxicol Pharmacol 2020;78:103411.

35. Emami A, Javanmardi F, Pirbonyeh N, et al. Prevalence of Underlying Diseases in Hospitalized Patients with COVID-19: a Systematic Review and Meta-Analysis. Arch Acad Emerg Med 2020;8:e35.

36. Lee AC, Chakladar J, Li WT, et al. Tobacco, but Not Nicotine and Flavor-less Electronic Cigarettes, Induces ACE2 and Immune Dysregulation. Int J Mol Sci 2020;21:1-16.

37. McAlinden KD, Eapen MS, Lu W, et al. COVID-19 and vaping: risk for increased susceptibility to SARS-CoV-2 infection? Eur Respir J 2020;56:2001645.

38. Majmundar A, Allem JP, Cruz TB, et al. Public Health Concerns and Unsubstantiated Claims at the Intersection of Vaping and COVID-19. Nicotine Tob Res 2020;22:1667-8.

39. Javelle E. Electronic cigarette and vaping should be discouraged during the new coronavirus SARS- CoV-2 pandemic. Arch Toxicol 2020;94:2261-2.

40. Gaiha SM, Cheng J, Halpern-Felsher B. Association 
Between Youth Smoking, Electronic Cigarette Use, and COVID-19. J Adolesc Health 2020;67:519-23.

41. The EVALI outbreak and vaping in the COVID-19 era. Lancet Respir Med 2020;8:831.

42. Shahab L, Goniewicz ML, Blount BC, et al. Nicotine, carcinogen, and toxin exposure in long-Term e-cigarette and nicotine replacement therapy users. Ann Intern Med 2017;166:390-400.

43. Goniewicz ML, Knysak J, Gawron M, et al. Levels of selected carcinogens and toxicants in vapor from electronic cigarettes. Tob Control 2014;23:133-9.

44. Fuller TW, Acharya AP, Meyyappan T, et al. Comparison of Bladder Carcinogens in the Urine of E-cigarette Users Versus Non E-cigarette Using Controls. Sci Rep 2018;8:507.

45. Letaiová $\mathrm{S}$, Medveová A, Ovíková A, et al. Bladder cancer, a review of the environmental risk factors. Environ Heal A Glob Access Sci Source 2012;11:1-5.

46. Carreón T, Hein MJ, Hanley KW, et al. Bladder cancer incidence among workers exposed to o-toluidine, aniline and nitrobenzene at a rubber chemical manufacturing plant. Occup Environ Med 2014;71:175-82.

47. Schaal CM, Bora-Singhal N, Kumar DM, et al. Regulation of Sox 2 and stemness by nicotine and electronic-cigarettes in non-small cell lung cancer. Mol Cancer 2018;17:149.

48. Tommasi S, Bates SE, Behar RZ, et al. Limited mutagenicity of electronic cigarettes in mouse or human cells in vitro. Lung Cancer 2017;112:41-6.

49. Zahedi A, Phandthong R, Chaili A, et al. Epithelial-tomesenchymal transition of A549 lung cancer cells exposed to electronic cigarettes. Lung Cancer 2018;122:224-33.

50. Avino P, Scungio M, Stabile L, et al. Second-hand aerosol from tobacco and electronic cigarettes: Evaluation of the smoker emission rates and doses and lung cancer risk of passive smokers and vapers. Sci Total Environ 2018;642:137-47.

51. Filippidis FT, Laverty AA, Mons U, et al. Changes in smoking cessation assistance in the European Union between 2012 and 2017: Pharmacotherapy versus counselling versus e-cigarettes. Tob Control 2019; 28:95-100.

52. Baldassarri SR, Bernstein SL, Chupp GL, et al. Electronic cigarettes for adults with tobacco dependence enrolled in a tobacco treatment program: A pilot study. Addict Behav 2018;80:1-5.

53. Patil S, Arakeri G, Patil S, et al. Are electronic nicotine delivery systems (ENDs) helping cigarette smokers quit?Current evidence. Journal of Oral Pathology and Medicine
2020;49:181-9.

54. Berry KM, Reynolds LM, Collins JM, et al. E-cigarette initiation and associated changes in smoking cessation and reduction: The Population Assessment of Tobacco and Health Study, 2013-2015. Tob Control 2019;28:42-9.

55. Kulik MC, Lisha NE, Glantz SA. E-cigarettes Associated With Depressed Smoking Cessation: A Cross-sectional Study of 28 European Union Countries. Am J Prev Med 2018;54:603-9.

56. Kalkhoran S, Glantz SA. E-cigarettes and smoking cessation in real-world and clinical settings: A systematic review and meta-analysis. Lancet Respir Med 2016; 4:116-28.

57. Hershberger AR, VanderVeen JD, Karyadi KA, et al. Transitioning From Cigarettes to Electronic Cigarettes Increases Alcohol Consumption. Subst Use Misuse 2016;51:1838-45.

58. Hajek P, Phillips-Waller A, Przulj D, et al. A randomized trial of E-cigarettes versus nicotine-replacement therapy. N Engl J Med 2019;380:629-37.

59. Cahill K, Stevens S, Perera R, et al. Pharmacological interventions for smoking cessation: An overview and network meta-analysis. Cochrane Database Syst Rev 2013;(5):CD009329.

60. Kadimpati S, Nolan M, Warner DO. Attitudes, Beliefs, and Practices Regarding Electronic Nicotine Delivery Systems in Patients Scheduled for Elective Surgery. Mayo Clin Proc 2015;90:71-6.

61. Nolan M, Leischow S, Croghan I, et al. Feasibility of Electronic Nicotine Delivery Systems in Surgical Patients. Nicotine Tob Res 2016;18:1757-62.

62. Luxton NA, Shih P, Rahman MA. Electronic Cigarettes and Smoking Cessation in the Perioperative Period of Cardiothoracic Surgery: Views of Australian Clinicians. Int J Environ Res Public Health 2018;15:2481.

63. Rau AS, Reinikovaite V, Schmidt EP, et al. Electronic cigarettes are as toxic to skin flap survival as tobacco cigarettes. Ann Plast Surg 2017;79:86-91.

64. Troiano C, Jaleel Z, Spiegel JH. Association of Electronic Cigarette Vaping and Cigarette Smoking With Decreased Random Flap Viability in Rats. JAMA Facial Plast Surg 2019;21:5-10.

65. Sørensen LT. Wound healing and infection in surgery: The pathophysiological impact of smoking, smoking cessation, and nicotine replacement therapy: A systematic review. Ann Surg 2012;255:1069-79.

66. Fracol M, Dorfman R, Janes L, et al. The Surgical Impact of E-Cigarettes: A Case Report and Review of the Current 
Literature. Arch Plast Surg 2017;44:477-81.

67. Krishnan NM, Han KD, Nahabedian MY. Can

E-cigarettes cause free flap failure? A case of arterial vasospasm induced by electronic cigarettes following microsurgical breast reconstruction. Plast Reconstr Surg Glob Open 2016;4:e596.

68. Pywell MJ, Wordsworth M, Kwasnicki RM, et al. The Effect of Electronic Cigarettes on Hand Microcirculation. J Hand Surg Am 2018;43:432-8.

69. Vishal Rao US, Arakeri G, Ravishankar S, et al. The

Cite this article as: Famiglietti A, Memoli JW, Khaitan PG. Are electronic cigarettes and vaping effective tools for smoking cessation? Limited evidence on surgical outcomes: a narrative review. J Thorac Dis 2021;13(1):384-395. doi: 10.21037/jtd-202529
E-cigarette ban in India-A step in the right direction? Journal of Oral Pathology and Medicine. 2020;49:617-20.

70. Goldberg RJ, Lapane K, Lemon S, et al. The Smoking Gun Can We Do for Gun Control What We Are Doing to Control the Vaping and E-Cigarettes Epidemic? Med Care 2020;58:1-3.

71. WHO Report on Global Tobacco Epidemic, 2019. World Health Organization [Internet]. 2019. Available online: https://www.who.int/tobacco/global_report/en/ 